\title{
INEFICIENCIA DE LA PULPA DE CITRUS SIN ADICIÓN NITROGENADA \\ PARA AUMENTAR EL PESO DE BOVINOS CRUZA CEBU EN CRECIMIEN- TO. EFECTOS SOBRE LOS ELECTROLITOS PLASMÁTICOS
}

\author{
Coppo, José A., Coppo, Norma B., Revidatti, María A., Capellari, Adriana y Navamuel, Juan M. \\ Cátedras de Fisiología y Zootecnia General, Facultad de Ciencias Veterinarias, UNNE. \\ E-mail: jcoppo@vet.unne.edu.ar
}

\section{RESUMEN}

La pulpa de citrus es un suplemento altamente energético, pero su contenido proteico es bajo. Por esta razón se recomienda adicionarle nitrógeno cuando se destina a la alimentación de bovinos en crecimiento. Dada la existencia de reportes aseverando que el ganado cebú efectuaría un mejor aprovechamiento de los nutrientes, el objetivo de este estudio fue indagar los efectos de dicho residuo agroindustrial, sin adición nitrogenada, sobre el peso y los electrolitos plasmáticos de animales cruza cebú en crecimiento. Quince vaquillonas (200-250 kg de peso) operaron como controles sobre pastura natural (C) y otras 15 fueron suplementadas (S) con pulpa fresca de citrus a razón del $0,7 \%$ del peso vivo, durante 3 meses (invierno). Ambos lotes fueron controlados a los 0, 30, 60 y 90 días, bajo un diseño de medidas repetidas. Los datos finales indicaron que en $\mathrm{S}$ ocurrieron disminuciones significativas $(\mathrm{p}<0,05)$ de fósforo inorgánico (3,29 versus 4,88 mg/dl en C), potasio $(4,03$ versus $4,39 \mathrm{meq} / \mathrm{l})$ y cobre $(30,4$ versus 71,6 ug/dl), así como aumentos significativos de hierro (138 versus $95 \mathrm{ug} / \mathrm{dl}$ ) y calcio $(10,8$ versus $9,21 \mathrm{mg} / \mathrm{dl})$. Los contrastes ortogonales revelaron que las diferencias entre $\mathrm{C}$ y $\mathrm{S}$ comenzaron a los 30 y 60 días, según el caso. Las variaciones de sodio, magnesio y peso $(\mathrm{S}=$ 60 versus $\mathrm{C}=57 \mathrm{~g} /$ animal $/$ día $)$ no fueron significativas. Se concluye que la pulpa de citrus, si bien elevó algunos indicadores bioquímicos del estado de nutrición mineral, fue inefectiva para aumentar significativamente el peso de los animales en crecimiento durante el déficit invernal de pasturas.

Palabras claves: pulpa de citrus, suplementación, vaquillonas cruza cebú, peso, electrolitos plasmáticos.

\begin{abstract}
SUMMARY
Citrus pulp is a highly energy supplement, but its protein content is low. For this reason it is advisable to add nitrogen when it is going to be used to feed growing cattle. Since that zebu cattle would make a better use of nutrients, according to some reports, the objective of the study was to investigate the effects of this agroindustrial residue on weight and plasma electrolytes from growing half-bred zebu animals, without nitrogen addition. Fifteen heifers (200-250 kg liveweight) operated as controls on natural pasture (C), and others 15 were supplemented (S) with fresh citrus pulp ( $0,7 \%$ liveweight $)$, during 3 months (winter). Both lots were controlled at 0 , 30, 60 and 90 days, by means of a repeated measures design. In $\mathrm{S}$, final data indicated significant decreases $(p<0,05)$ of inorganic phosphorous $(3,29$ versus $4,88 \mathrm{mg} / \mathrm{dl}$ in $\mathrm{C})$, potassium (4,03 versus $4,39 \mathrm{meq} / \mathrm{l})$ and copper $(30,4$ versus $71,6 \mathrm{ug} / \mathrm{dl})$, as well as significant increases of iron (138 versus $95 \mathrm{ug} / \mathrm{dl})$ and calcium (10,8 versus 9,21 $\mathrm{mg} / \mathrm{dl})$. Orthogonal contrasts revealed that differences between $\mathrm{C}$ and $\mathrm{S}$ began at 30 and 60 days, depending on the case. Sodium, magnesium and weight variations ( $\mathrm{S}=60$ versus $\mathrm{C}=57 \mathrm{~g} /$ animal/day) were not significant. In conclusion, citrus pulp increased some biochemical indicators of mineral nutrition state, but it was ineffective to increase significantly the weight of growing animals during the winter pasture deficit.
\end{abstract}

Key words: citrus pulp, winter supplement, halfbred zebu heifers, liveweight, plasma electrolytes. 


\section{INTRODUCCIÓN}

La pulpa de citrus es el subproducto de la industria de los jugos homónimos. Constituye un suplemento alimentario de alto valor energético para los rumiantes (Peruchena, 1992). Posee elevado contenido de glúcidos, como azúcares y pectinas, pero su contenido proteico es bajo (Brown, 1990; Hofer et al., 1991; Ammerman y Henry, 1992; Kuvera et al., 1993). Cuanto más semillas contenga, tanto más alta será su proporción de nitrógeno y lípidos (Martínez y Fernández, 1980).

Con relación al pastizal natural, la pulpa de citrus posee menor proporción de fibra detergente neutro $(25,4$ versus $67,2 \%)$, similar contenido de materia orgánica $(91,5$ versus $89.9 \%$ ) y mayor digestibilidad in vitro de dicha materia orgánica $(89,6$ versus 55,9\%) (Hofer y Galli, 1991). La digestibilidad de materia seca y proteínas es alta (92 y 65\% respectivamente), por lo cual fermenta rápidamente en el rumen (Aguilera, 1989; Kuvera et al., 1993).

Las gramíneas subtropicales existentes en el nordeste argentino son de bajo valor nutritivo, poseen alto porcentaje de materia seca y fibra, así como bajos niveles de energía y proteínas. En estas condiciones, la cría extensiva de bovinos para carne ostenta una productividad de 15 a 50 $\mathrm{kg} / \mathrm{ha} / \mathrm{año}$, según áreas, siendo la carga/ha de 1,5 a $4,0 \mathrm{ha} / \mathrm{vaca}$, el porcentaje de destete es de 40 $50 \%$ (con pesos de 140 a $160 \mathrm{~kg}$ ), el entore de las vaquillas ocurre a los 3-4 años y la terminación de novillos suele requerir 4-6 años (Peruchena, 1992).

Dicha zona es considerada como un área marginal de producción ganadera, predominando el ganado resultante del cruzamiento de bovinos europeos y criollos con razas cebuínas, los cuales se crían generalmente sobre pastizales naturales. Debido al déficit invernal de los pastizales, los vacunos pueden llegar a perder parte del peso obtenido durante el resto de las temporadas (Arias, 1991). Esta circunstancia puede subsanarse administrando suplementos alimentarios, entre los cuales se cuenta la pulpa de citrus, dado que en la zona existen numerosas plantaciones de citrus, así como plantas elaboradoras de jugos. No se hallaron reportes que relacionen el consumo de pulpa "fresca" de citrus, utilizada como suplemento único, con indicadores de crecimiento y nutrición mineral en vaquillonas cruza cebú de cría extensiva, en el nordeste argentino.

El objetivo de este estudio fue investigar si la suplementación invernal con pulpa fresca de citrus, sin adición nitrogenada, era capaz de incrementar el peso de bovinos cruza cebú en etapa de crecimiento, así como indagar sus efectos sobre los niveles de electrolitos plasmáticos.

\section{MATERIALES Y MÉTODOS}

Los ensayos se llevaron a cabo en el Departamento Bella Vista, Provincia de Corrientes, Argentina. Se utilizaron 30 vaquillonas de recría (cruza Cebú x Hereford) de 16-18 meses de edad, clínicamente sanas, mantenidas en potreros $(0,5 \mathrm{EV} / \mathrm{ha})$ con homogénea cantidad y calidad de pastura natural $(2000 \mathrm{~kg} / \mathrm{MS} / \mathrm{ha})$, donde los niveles de proteína cruda usualmente son del 4$6 \%$, con $\mathrm{EM}=1,7-1,8 \mathrm{Mcal} / \mathrm{kg}$ MS (Peruchena 1992). Empleando un diseño experimental completamente aleatorizado, 15 animales fueron suplementados durante 3 meses (invierno) con pulpa fresca de citrus, suministrada a razón del $0,7 \%$ del peso vivo promedio (grupo suplementado, S), mientras que los 15 restantes no recibieron suplementación (grupo control, C).

La materia seca de la pulpa de citrus suministrada $(\mathrm{MS}=15,86 \%)$ contenía $5,4 \%$ de proteína bruta, $19 \%$ de fibra cruda, $2,7 \%$ de grasa bruta, $4,2 \%$ de cenizas, $68,65 \%$ de extracto no nitrogenado, $0,17 \%$ de fósforo, $0,54 \%$ de calcio, $0,03 \%$ de sodio, $0,50 \%$ de potasio, $725 \mathrm{mg} / \mathrm{kg}$ de magnesio, $15 \mathrm{mg} / \mathrm{kg}$ de manganeso, $78 \mathrm{mg} / \mathrm{kg}$ de cinc, $83 \mathrm{mg} / \mathrm{kg}$ de hierro y $15 \mathrm{mg} / \mathrm{kg}$ de cobre, con 3,62 Mcal $/ \mathrm{kg} \mathrm{MS}$.

Ambos grupos fueron sometidos a pesajes y extracciones de sangre por venopunción yugular, a los $0,30,60$ y 90 días. Los pesos se obtuvieron individualmente en una báscula, previo encierro nocturno. La sangre fue centrifugada $(700 \mathrm{~g}, 10$ min) para obtener suero, el cual se preservó a $4^{\circ} \mathrm{C}$ hasta ser analizado. Las pruebas de laboratorio se llevaron a cabo mediante técnicas convencionales (Coles 1989). Mediante un espectrofotómetro Labora Mannheim 4010 UV-visible, se efectuaron las determinaciones de magnesio (técnica de la calmagita, lecturas a $520 \mathrm{~nm}$, reactivos Randox), calcio (cresolftaleincomplexona, $578 \mathrm{~nm}$, GT-Lab), fósforo inorgánico (fosfomolibdato, $620 \mathrm{~nm}$, Wiener Lab), hierro (PBTS, 560 $\mathrm{nm}$, Wiener Lab) y cobre (batocuproína, $436 \mathrm{~nm}$, Boehringer). Sodio y potasio fueron evaluados en un fotómetro de llama Metrolab 505, con reactivos Biopur.

Estadísticamente, la homogeneidad inicial fue corroborada por superposición de intervalos de confianza (IC $\pm 95 \%$ ) y la normalidad distributiva fue verificada por la prueba de Wilk-Shapiro (WS). Las estadísticas descriptivas paramétricas incluyeron indicadores de tendencia central (media aritmética, $\xi$ ) y dispersión (desvío estándar, DE). El análisis de la variancia para medidas repetidas (Anova), incluyó la significación estadística para los efectos tratamiento (suplementación) y tiempo (crecimiento), así como la interacción entre ambos. Con el objeto de determinar el momento en que las diferencias entre $\mathrm{C}$ y $\mathrm{S}$ 
comenzaron a ser significativas (p), la comparación de medias post-Anova se realizó mediante contrastes ortogonales. Para todas las inferencias fue fijado un $\alpha=5 \%$, por debajo del cual se rechazó la hipótesis nula de igualdad.

\section{RESULTADOS Y DISCUSIÓN}

Los valores iniciales de peso y concentraciones electrolíticas fueron homogéneos (IC $\pm 95 \%$ ) y se distribuyeron en forma simétrica (WS). El Anova de medidas repetidas no detectó interacciones entre los efectos tiempo y tratamiento. Los niveles plasmáticos estudiados encuadraron en el intervalo de referencia regional para la edad y tipo de ganado (Coppo, 2001). Los valores medios obtenidos, así como su dispersión, son expuestos en Tabla 1.

Al cabo de los 90 días de estudio, los aumentos de peso fueron escasos tanto en $\mathrm{C}(5,2 \mathrm{~kg})$ como en $\mathrm{S}(5,4 \mathrm{~kg})$, resultando ganancias de $57,7 \mathrm{y}$ $60,0 \mathrm{~g} /$ animal/día respectivamente. En el nordeste argentino es habitual que durante el invierno disminuya la velocidad de crecimiento e incluso que los bovinos pierdan peso, debido a la declinación de la cantidad y calidad de las pasturas naturales (Arias, 1991; Peruchena, 1992). Resulta evidente que la pulpa fresca de citrus, rica en energía pero escasa en contenido proteico, fue incapaz de lograr que los animales suplementados superaran significativamente el peso de los controles. Los contrastes ortogonales revelaron que las diferencias entre $\mathrm{C}$ y S solo fueron signi- ficativas $(\mathrm{p}<0,05)$ a los 30 días de iniciada la suplementación, como muestra la Figura 1.

Por ser un suplemento muy voluminoso (Sablich, 1997) y rápidamente fermentescible (Ammerman y Henry, 1992; Alfaro, 1992), no se descarta que la pulpa de citrus haya disminuido el consumo de pastura en los animales del lote $\mathrm{S}$, limitando su velocidad de crecimiento. Garciarena et al. (1997) demostraron que en novillos el consumo de alimento disminuía a medida que aumentaba la proporción de pulpa de citrus en la dieta, circunstancia que se atribuyó a la alta degradabilidad del suplemento (INTA, 1997).

Se afirma que la exigua proporción proteica de este residuo agroindustrial resulta insuficiente para mantener la síntesis ruminal de proteína microbiana en ganado lechero (Kuvera et al., 1993). El ganado cruza cebú se caracterizaría por poseer mayor eficiencia metabólica que los bovinos europeos, al lograr "extraer mayor cantidad de principios nutritivos a partir de un menor volumen de alimento", por lo cual estaría mejor capacitado para aprovechar los pastos duros y escasos, así como para soportar épocas de sequía (Inchausti y Tagle, 1980). Pese a que en condiciones pastoriles desfavorables, las cruzas índicas obtendrían mayores pesos que las razas británicas debido a que efectúan un mejor aprovechamiento de los nutrientes (Howes, 1989), en el presente estudio tal circunstancia no se tradujo en mayor velocidad de crecimiento.

Tabla 1. Valores obtenidos $(\xi \pm \mathrm{DE})$ en animales controles (C) y suplementados (S).

\begin{tabular}{lllll}
\hline Parámetro & \multicolumn{2}{l}{ Valores iniciales (día 0) } & \multicolumn{2}{l}{ Valores finales (día 90) } \\
\cline { 2 - 5 } & $\mathrm{C}(\mathrm{n}=15)$ & $\mathrm{S}(\mathrm{n}=15)$ & $\mathrm{C}(\mathrm{n}=15)$ & $\mathrm{S}(\mathrm{n}=15)$ \\
\hline Peso vivo $(\mathrm{kg})$ & $227,6 \pm 23,2 \mathrm{a}$ & $228,7 \pm 24,6 \mathrm{a}$ & $232,8 \pm 25,3 \mathrm{~b}$ & $234,1 \pm 25,9 \mathrm{~b}$ \\
$\mathrm{Ca}(\mathrm{mg} / \mathrm{dl})$ & $9,56 \pm 0,7 \mathrm{a}$ & $9,72 \pm 0,8 \mathrm{a}$ & $9,21 \pm 0,5 \mathrm{a}$ & $10,8 \pm 0.9 \mathrm{~b}$ \\
$\mathrm{P}(\mathrm{mg} / \mathrm{dl})$ & $5,90 \pm 1,2 \mathrm{a}$ & $5,72 \pm 1,1 \mathrm{a}$ & $4,88 \pm 0.9 \mathrm{~b}$ & $3,29 \pm 0,7 \mathrm{c}$ \\
$\mathrm{Mg}(\mathrm{mg} / \mathrm{dl})$ & $2,38 \pm 0,2 \mathrm{a}$ & $2,43 \pm 0,3 \mathrm{a}$ & $2,29 \pm 0,2 \mathrm{a}$ & $2,55 \pm 0,4 \mathrm{a}$ \\
$\mathrm{Na}(\mathrm{meq} / \mathrm{l})$ & $139 \pm 5 \mathrm{a}$ & $141 \pm 7 \mathrm{a}$ & $137 \pm 4 \mathrm{a}$ & $140 \pm 5 \mathrm{a}$ \\
$\mathrm{K}(\mathrm{meq} / \mathrm{l})$ & $4,42 \pm 0,5 \mathrm{a}$ & $4,35 \pm 0,4 \mathrm{a}$ & $4,39 \pm 0,5 \mathrm{a}$ & $4,03 \pm 0,3 \mathrm{~b}$ \\
$\mathrm{Fe}(\mathrm{ug} / \mathrm{dl})$ & $109 \pm 17 \mathrm{a}$ & $112 \pm 19 \mathrm{a}$ & $95 \pm 15 \mathrm{~b}$ & $138 \pm 21 \mathrm{c}$ \\
$\mathrm{Cu}(\mathrm{ug} / \mathrm{dl})$ & $80,5 \pm 9,2 \mathrm{a}$ & $77,2 \pm 7,5 \mathrm{a}$ & $71,6 \pm 7,1 \mathrm{~b}$ & $30,4 \pm 5,3 \mathrm{c}$ \\
\hline
\end{tabular}

En cada fila, letras distintas indican diferencias significativas entre conjuntos de medias $(\mathrm{p}<0,05)$. 


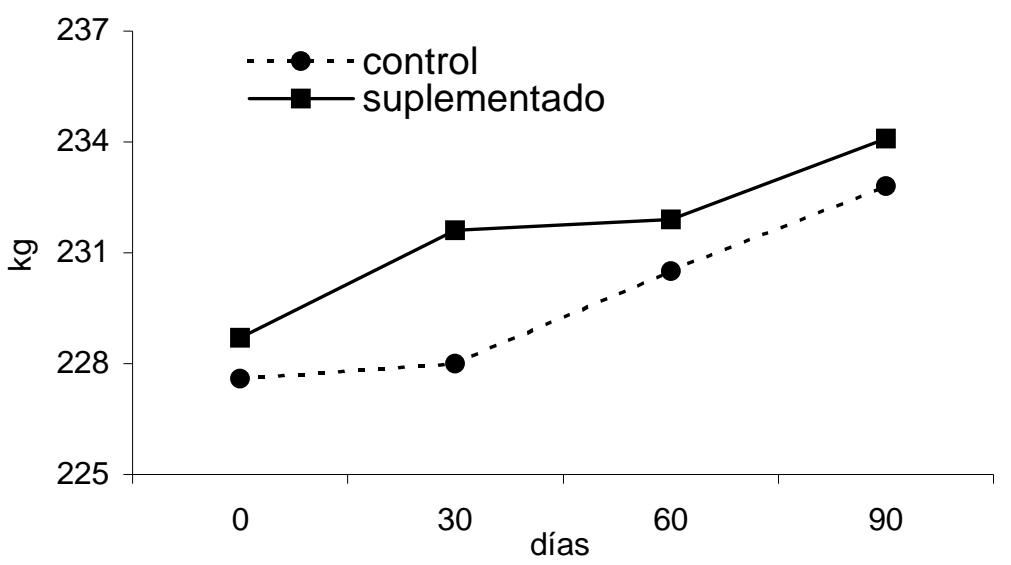

Figura 1. Evolución del peso vivo en lotes control y suplementado.

Los resultados obtenidos avalan la aseveración que para el ganado en crecimiento, la suplementación con pulpa de citrus requeriría la adición de proteína o nitrógeno no proteico (Brown, 1990; Hofer et al., 1991; Kuvera et al., 1993). Al ser suministrada a novillos $(6 \mathrm{~kg} /$ animal/día durante 3 meses) con adición de urea, la pulpa de citrus generó ganancias de peso del orden de 500 g/animal/día (Carnevali et al., 1973).

Para engordar ganado adulto no sería necesaria la adición nitrogenada. Anteriormente, este grupo de trabajo constató ganancias de peso de 612 g/animal/día (versus $372 \mathrm{~g} / \mathrm{animal} /$ día en los controles), en vacas de invernada cruza cebú suplementadas con pulpa fresca de citrus durante el invierno (Navamuel et al., 2002).

En la Tabla 2 se consignan los datos arrojados por el análisis de la variancia de medidas repetidas, surgiendo que si bien el efecto tiempo fue significativo para el peso de los animales (crecimiento), el efecto tratamiento (suplementación) no fue significativo. En cuanto a los electrolitos plasmáticos, se constató significatividad del efecto tiempo para las disminuciones de fósforo inorgánico, potasio y cobre (y no significativas para las declinaciones de magnesio y sodio), que se atribuyen tanto al empobrecimiento invernal de las pasturas (Mufarrege, 1993; Peruchena, 1992) como a razones ontogénicas (Coppo, 2001).

En S, la significatividad de los aumentos de hierro y calcio deberían atribuirse al aporte efectuado por la pulpa de citrus, rica en dichos minerales (Cunha, 1973; Morrison, 1980; Brown, 1990). El contenido de magnesio también sería alto en dicho residuo agroindustrial (Cunha, 1973), pese a lo cual el aumento de este electrolito, al igual que la elevación del sodio, no fueron significativos en $\mathrm{S}$. Las disminuciones de fósforo inorgánico y cobre en $\mathrm{S}$ podrían atribuirse, además del menor consumo total de materia seca citado precedentemente (Garciarena et al., 1997), a la insuficiente proporción de estos minerales en el suplemento administrado (Brown, 1990; Chapman et al., 1972; Morrison, 1980). Se afirma que, a excepción del hierro, la proporción de oligoelementos sería escasa en los residuos cítricos (Chapman et al., 1972), razón por la cual deberían adicionársele minerales para lograr significativos aumentos de peso (Kirk y Davis, 1954).

Tabla 2. Resultados del Anova de medidas repetidas.

\begin{tabular}{llll}
\hline parámetro & efecto tiempo $(\mathrm{C}+\mathrm{S})$ & efecto tratamiento $(\mathrm{S})$ & día \\
\hline Peso vivo & aumento $*$ & aumento (NS) & - \\
$\mathrm{Ca}$ & aumento (NS) & aumento * & 60 \\
$\mathrm{P}$ & disminución $*$ & disminución $*$ & 30 \\
$\mathrm{Mg}$ & disminución (NS) & aumento (NS) & - \\
$\mathrm{Na}$ & disminución (NS) & aumento (NS) & - \\
$\mathrm{K}$ & disminución* & disminución * & 60 \\
$\mathrm{Fe}$ & aumento (NS) & aumento * & 30 \\
$\mathrm{Cu}$ & disminución * & disminución * & 30 \\
\hline
\end{tabular}

C: lote control, $\mathrm{S}$ : lote suplementado, día: inicio de las diferencias significativas entre $\mathrm{C} \mathrm{y} \mathrm{S,}{ }^{*} \mathrm{p}<0,05$, NS: no significativo. 
El potasio disminuyó significativamente en el lote suplementado, pese a que la pulpa de citrus poseería elevados niveles de este mineral (Cunha, 1973). La razón de esta incongruencia quizás resida en el hecho que la alta disponibilidad de energía (glúcidos) en períodos de baja exigencia metabólica (ausencia de gestación y lactación), provoca mayor liberación de insulina, la cual es capaz de generar hipokalemia al aumentar la actividad de la bomba $\mathrm{Na}^{+}-\mathrm{K}^{+}$-ATPasa, por medio de la cual el potasio ingresa a las células y consecuentemente disminuye su concentración en los líquidos extracelulares y el plasma (Ganong, 1996; Cirio y Tebot, 2000).

\section{CONCLUSIONES}

La pulpa fresca de citrus suministrada durante el invierno a bovinos cruza cebú en crecimiento, si bien elevó significativamente los niveles plasmáticos de hierro y calcio, así como no significativamente los de sodio y magnesio, produjo disminuciones significativas de cobre, potasio y fósforo inorgánico, y fue ineficaz para aumentar significativamente el peso vivo, con relación al alcanzado por animales de similares características mantenidos sobre pastura natural sin suplementación.

\section{BIBLIOGRAFÍA}

Aguilera, J.F. 1989. Aprovechamiento de subproductos agroindustriales en la alimentación de rumiantes. Prod. Anim. 9: 253-267.

Alfaro, M.A. 1992. Sustitución parcial del grano de sorgo por pulpa deshidratada de naranja en novillos engordados a corral. Tesis de Maestría en Ciencias, Facultad de Agronomía, Universidad de Tamaulipas, México, 35 pp.

Ammerman, C.B.; Henry, P.R. 1992. Utilización de subproductos cítricos para ganado. Memorias de la Conferencia Internacional sobre Ganadería Tropical, Universidad de Florida, U.S.A., p. 66-73.

Arias, A.A. 1991. Suplementación con subproductos energéticos proteicos de vacunos para carne en pastoreo en la Provincia de Corrientes. Informe INTA EEA-Corrientes, 13 pp.

Brown, W.F. 1990. Wet and dry citrus pulp are both good feed for cattle. Florida Cattle \& Livestock J. 12: 29-34.

Carnevali, A.A.; Chicco, C.F.; Verde, G. 1973. Utilización de altos niveles de pulpa de cítricos y de urea en raciones de engorde para bovinos. Agron. Trop. 22: 261-269.

Cirio, A.; Tebot, I. 2000. Fisiología Metabólica de los Rumiantes, Ed. CSIC, Montevideo, 146 pp.

Coles, E.H. 1989. Veterinary Clinical Pathology, $4^{\text {th }}$ ed., Saunders, Philadelphia, 486 pp.

Coppo, J.A. 2001. Fisiología del Medio Interno, Ed. Dunken, Buenos Aires, $297 \mathrm{pp}$

Cunha, T.J. 1973. Naranjas para el ganado. Agric. Amer. 10: 54-56.
Chapman, H.L.; Ammerman, C.B.; Baker, F.S.; Hentges, J.F.; Hayes, B.W.; Cunha, T.J. 1972. Citrus feeds for beef cattle. Agric. Expt. Stat. Florida Univ. Bull. 751: 1-34.

Ganong, W.F. 1996. Review of Medical Physiology, 15th ed., Appleton \& Lange, Philadelphia, 962 pp.

Garciarena, A.D.; Hofer, C.C.; Christiansen, A.R. 1997. Consumo, dinámica y ambiente de dietas con alto contenido de pulpa de citrus. Prod. Anim. 17: 2-3.

Hofer, C.C.; Galli, I.O. 1991. Pulpa de citrus en dietas para terneros destetados precozmente. Prod. Anim. INTA, Informe Técnico N 3: 112-116.

Hofer, C.C.; Pozzolo, O.R.; Galli, I.O. 1991. Evaluación in vitro del silaje de pulpa de citrus para la alimentación de rumiantes. Prod. Anim. INTA, Informe Técnico N 3: 102-104.

Howes, J.R. 1989. Potencial digestivo del Brahman comparado con el de Hereford. Cebú 36: 36-38.

Inchausti, D.; Tagle, E.C. 1980. Bovinotecnia, $6^{\circ}$ ed., El Ateneo, Buenos Aires, 800 pp.

INTA EEA-Concepción del Uruguay. 1997. Suplementación a terneras con pulpa de citrus y grano de sorgo. Efecto sobre el consumo voluntario de heno. Memorias de Jornada Anual de Difusión Técnica sobre Alimentación con Subproductos en Sistemas de Producción de Carne y Leche, p. 5657.

Kirk, W.G.; Davis, G.K. 1954. Citrus products for beef cattle. Agric. Expt. Stat. Florida Univ. Bull. 538: 12-19.

Kuvera, J.C.; Nazar Balboa, H.; Alfaro Ramos, M.A. 1993. Utilización de la pulpa deshidratada de cítricos en la alimentación de los rumiantes. Biotam 5: $1-5$.

Martínez, P.J.; Fernández, C.J. 1980. Composition of citrus pulp. Anim. Fed. Sci \&\& Technol. 5: 1-10.

Morrison, F.B. 1980. Alimentos y Alimentación del Ganado, $21^{\circ}$ ed., UTEHA, México, 588 pp.

Mufarrege, D. 1993. Distribución estacional de nutrientes para el ganado en pastizales del nordeste argentino. Informe Anual del Instituto Nacional de Tecnología Agropecuaria, EEA Mercedes, Corrientes, p. 102-107.

Navamuel, J.M.; Fioranelli, S.A.; Capellari, A.; Revidatti, M.A.; Coppo, N.B.; Coppo, J.A. 2002. Weight gain of wintering cows supplemented with citrus pulp. Livestock Research 14: 28-32.

Peruchena, C.O. 1992. Nutrición de bovinos sobre pastizales de baja calidad del NEA. Anales de la XII Sesión de Comunicaciones Científicas de la Facultad de Ciencias Veterinarias UNNE, Corrientes, p. 17-22.

Sablich, J. 1997. Residuos de la industria citrícola en la alimentación animal. Anales II Jornadas de Producción Animal, Facultad de Ciencias Veterinarias UNNE, Corrientes, p. 1-6. 